\title{
Higher BMI is linked to an increased risk of heart attacks in European adults: a Mendelian randomisation study
}

\author{
Benjamin Adams, Lauren Jacocks and Hui Guo (1)
}

\begin{abstract}
Background: BMI has been implicated as a risk factor for heart disease as a whole in multiple studies. Heart attack is one of the common complications of this disease. The aim of this study is to explore if elevated level of BMl causes an increase in the risk of heart attacks.

Methods: We used two Mendelian randomisation (MR) methods: inverse variance weighted estimation and robust adjusted profile score (RAPS) on the basis of summary data of adulthood BMI from Genetic Investigation of Anthropometric Traits consortium and heart attack data from the UK Biobank. BMI associated single nucleotide polymorphisms (SNPs) were used as instrumental variables.

Results: Seventy-two independent SNPs were associated with BMI $\left(P<5 \times 10^{-8}\right)$. Using these SNPs as instruments, $\mathrm{BMI}$ was found to be causally associated with heart attacks in inverse variance weighted MR analysis. The risk of heart attacks increased by $0.8 \%$ per 1 -SD $\left(\right.$ or $\left.4.5 \mathrm{~kg} / \mathrm{m}^{2}\right)$ increase in $\mathrm{BMI}(\mathrm{OR}=1.008$ with $95 \% \mathrm{Cl}(1.003,1.012), P=$ 0.001). RAPS provided concordant results ( $\mathrm{OR}=1.007$ with $95 \% \mathrm{Cl}(1.002,1.012), P=0.004)$.

Conclusions: This current study is the first to use MR to investigate causal relationship between BMI and heart attacks. Our findings suggest that high level of BMI may cause increased risk of heart attacks.
\end{abstract}

Keywords: BMI, Heart attacks, Causal relationship, Mendelian randomisation

\section{Background}

\section{The obesity pandemic}

It is no secret that being overweight is increasingly becoming the norm, with the latest research briefing in the houses of parliament claiming that $28.7 \%$ of adults in the UK are obese and a further $35.6 \%$ are overweight [1]. Obesity measured as a BMI $>30 \mathrm{~kg} / \mathrm{m}^{2}$ has been linked to a plethora of ailments through observational studies [2-6]. According to the Office for National Statistics, in 2018, the leading cause of deaths in the UK was

\footnotetext{
* Correspondence: hui.quo@manchester.ac.uk

Centre for Biostatistics, Division of Population Health, Health Services Research and Primary Care, Faculty of Medicine, Biology and Health, The University of Manchester, Manchester, UK
}

ischaemic heart disease for men, accounting for $13.2 \%$ of deaths [7].

BMI has been implicated as a risk factor for heart disease as a whole [8]. Many studies, however, only seem to tackle more generalised terms of the disease. A previous observational study has identified a link between heart attacks and BMI [4]. They observed a cohort of 899 obese individuals in adults between 35 and 74. After 10 years of observations, the study concluded that obesity was not an independent cardiovascular risk factor. This study is statistically underpowered due to small sample size. Furthermore, like other observational studies, an obvious issue is that it may suffer from problems of confounding (e.g. smoking, alcohol abuse) [9] and other sources of bias [10]. Conclusions from these studies have

(c) The Author(s). 2020 Open Access This article is licensed under a Creative Commons Attribution 4.0 International License, which permits use, sharing, adaptation, distribution and reproduction in any medium or format, as long as you give appropriate credit to the original author(s) and the source, provide a link to the Creative Commons licence, and indicate if changes were made. The images or other third party material in this article are included in the article's Creative Commons licence, unless indicated otherwise in a credit line to the material. If material is not included in the article's Creative Commons licence and your intended use is not permitted by statutory regulation or exceeds the permitted use, you will need to obtain permission directly from the copyright holder. To view a copy of this licence, visit http://creativecommons.org/licenses/by/4.0/ The Creative Commons Public Domain Dedication waiver (http://creativecommons.org/publicdomain/zero/1.0/) applies to the data made available in this article, unless otherwise stated in a credit line to the data. 
limited use as clinical development of treatments requires well-targeted causal factors [11-13]. In our study, we can put this statement to the test as we employ the use of Mendelian randomisation (MR) to bypass these issues, with the aim to better infer causality of obesity on heart attacks in a population of white European individuals.

\section{Methods}

\section{Study design}

In our investigation, we have applied two-sample MR to explore if an elevated level of BMI (regarded as exposure) causes increased risk of heart attacks (regarded as outcome) using BMI associated single nucleotide polymorphisms (SNPs) as the instruments.

Two-sample MR requires participants from two separate studies - one for exposure and the other for outcome, where the individuals do not overlap since overlapping data sets would lead to our results suffering from inflated type 1 error rates [14]. However, the two samples must be representative of the same population. Thus, we have used summary data of European descendants from two independent studies: Genetic Investigation of Anthropometric Traits (GIANT) consortium (for BMI) [15] and the UK Biobank (for heart attacks) [16].

\section{BMI data - GIANT}

The genetic instruments were SNPs selected from GIANT [15]. This study consists of a meta-analysis of a population from European ancestry containing 322,154 individuals from 114 studies. Summary level data was extracted (see Summary Data section for more details) for BMI associated SNPs $\left(P<5 \times 10^{-8}\right)$. These SNPs were further filtered by clumping carried out using the MR-Base platform [17] to ensure that the final set of instruments in MR analysis were independent of one another. Essentially the SNPs in linkage disequilibrium (LD) $\left(R^{2} \geq 0.001\right)$ were clumped together with only the SNP with the lowest $p$-value being retained.

\section{Heart attack data - UK biobank}

The UK Biobank data contains approximately half a million individuals aged between 40 and 69 years [18]. The participants were recruited from across the UK between 2006 and 2010 and asked to provide information via questionnaires, interviews, anthropometric measures and samples (e.g. blood, urine and saliva). The summary data we used for heart attacks was from GWAS results by the Neale lab who carried out rigorous quality control (QC) checks [16]. These checks whittled down the individuals involved to only QC positives $(n=337,199)$. The filter which caused the largest reduction in participants was the restriction to white British genetic ancestry only. Participants were also removed if they were closely related to other individuals in the study or had sex chromosome abnormalities. To learn more about the QC process please see the Neale Lab website [16].

As of 2018, over 92 million autosomal SNPs (directly genotyped or imputed) were available for analysis. All these SNPs were further restricted by minor allele frequency $\quad(\mathrm{MAF})>0.1 \%$, Hardy-Weinberg Equilibrium (HWE) $p$-value $>1 \times 10^{-10}$ in the QC positive individuals and an imputation score INFO $>0.8$ leaving approximately 13.8 million SNPs for analysis [16].

For the heart attack data obtained from the UK Biobank, participants were asked in a survey on their medical history to categorically state if they had had a doctor diagnosed heart attack or stroke, or suffered from angina or high blood pressure [19]. Participants could also state if they had suffered from none of the above. The data from this survey was converted into binary (1: suffered from a doctor diagnosed heart attack, 0 otherwise).

\section{Summary data}

Instead of using individual-level data, one of the advantages of MR analysis is leverage summary statistics at the SNP level (estimated SNP effects, standard errors and corresponding $p$-values from regression models, effect alleles and other alleles along with their frequencies). These summary statistics from many large-scale genome wide association studies (GWAS) are now made publicly available.

Summary data of BMI and heart attacks were extracted separately, for BMI associated SNPs, from the GIANT and UK Biobank studies [15, 16]. In GIANT, data were standardised such that per unit change in BMI corresponds to 1 standard deviation (or $4.5 \mathrm{~kg} / \mathrm{m}^{2}$ ) change in BMI. We then carried out harmonisation using the TwoSample MR package in $\mathrm{R}$ to make sure that the effects of a SNP on the outcome and exposure were relative to the same allele, which produced one merged dataset for our MR analysis.

\section{Statistical analysis}

Before the advent of MR, observational studies were greatly limited by problems of unobserved confounding. These limitations rendered many findings lack of causal interpretations. MR, however, circumvents these difficulties by mimicking randomised controlled experiment and assuming that the instruments (SNPs) fulfil three criteria listed in Fig. 1.

We used two MR methods: inverse variance weighted (IVW) estimation and MR robust adjusted profile score (RAPS) to estimate causal effect of BMI on heart attacks (log odds ratio), its standard error and corresponding $p$ value $[20,21]$. Both of the methods require that instrumental SNPs are associated with the exposure and 


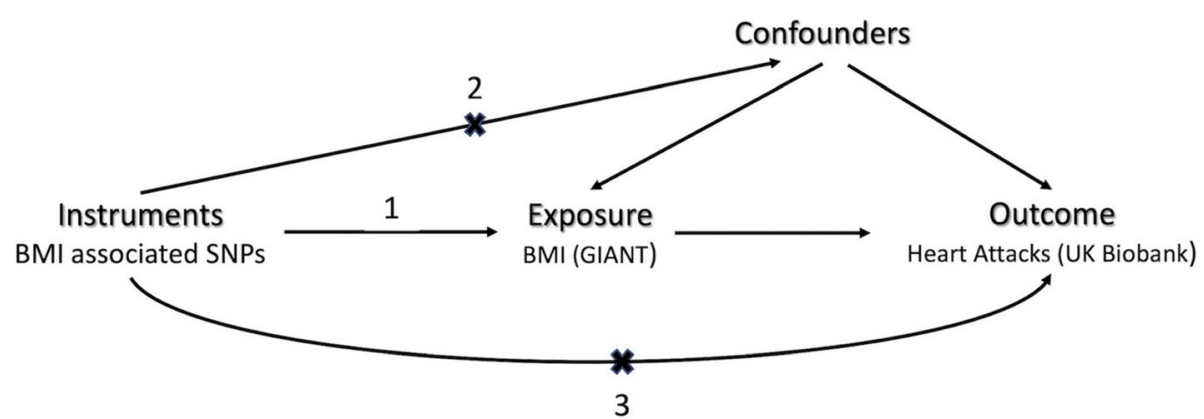

Fig. 1 Schematic representation of assumptions of our MR analysis. In this investigation, MR has been used to test the causal relationship between BMI and heart attacks in adulthood. The numbers indicate the three assumptions: 1) instrumental SNPs are associated with BMI; 2) instrumental SNPs are not associated with confounders; 3 ) instrumental SNPs do not have direct effects on the risk of heart attacks, i.e., their effects on heart attacks are mediated only through BMl

mutually independent. In IVW estimation, causal effect of an exposure on an outcome is first estimated as the ratio of the SNP-outcome association estimate to the SNP-exposure association estimate for each instrumental SNP. The IVW estimate is weighted average of these estimated causal effects, where each of the estimates is weighted by the inverse of their variance [22]. An obvious advantage of IVW is its use of multiple instruments to improve statistical power. However, it is prone to bias due to weak instruments. RAPS enhances the IVW estimation by replacing each weight with a function form of the causal effect and the variation of the instrument strength [23]. This approach provides more robust results in the presence of weak instruments than IVW estimate.

\section{Results}

Higher BMI causes increased risk of heart attacks in European descendants

Seventy-three independent SNPs that are at least 10,000 $\mathrm{kb}$ apart were found associated with BMI (Table 1). In our MR analysis we identified an outlier. The estimated causal effect of BMI on heart attacks obtained based on this SNP was more than 3 standard deviations away from the average causal effect calculated from all the 73 SNPs. We researched that particular outlier rs2075650 (located at bottom left, left panel of Fig. 2) in order to determine if it exhibits pleiotropy - it also influences other health conditions apart from heart attacks. According to the Ensembl database, this SNP is located in the TOMM40 gene and in close proximity to the $A P O E$ gene but may also influence Alzheimer's disease and age-related macular degeneration [24].

Both TOMM40 and APOE are located at $19 \mathrm{q} 13.32$ in the genome in chromosome 19 [25]. The protein encoded by TOMM40 is a channel-forming subunit of the translocase of the mitochondrial outer membrane. It is essential for the import of protein precursors into the mitochondria. The protein APOE, coding for apolipoprotein E, binds to fats in the blood to form lipoproteins. This allows for the transport of fats throughout the bloodstream, preventing the build-up of fats in the blood that could otherwise contribute to heart disease phenotypes if left there. Both of the genes have been linked to white matter integrity in the brain and the onset of Alzheimer's disease, although nothing has been conclusively proven [26].

We re-ran MR by excluding the outlier rs2075650 as a sensitivity analysis. Although this SNP might introduce pleiotropy, our MR results are concordant, with or without rs2075650, as shown in the two panels of Fig. 2. The slopes of the lines represent estimated log odds ratios. Both of the MR methods (IVW in red, RAPS in blue) have shown evidence for a positive causal effect of BMI on heart attacks. For simplicity, we only report estimated causal effect (odds ratio) from MR using the remaining 72 instruments in Table 2.

Table 2 lists results from the two MR methods: inverse variance weighted (IVW) estimation and robust adjusted profile score (RAPS) using the 72 independent BMIassociated SNPs as instruments. Estimated OR: estimated odds ratio. It states the estimated change in the risk of having a heart attack, for every $4.5 \mathrm{~kg}$ increase of weight per square metre. 95\% CI: 95\% confidence interval.

The risk of heart attacks increased by $0.8 \%$ (in IVW) and $0.7 \%$ (in RAPS) per 1 -SD (or $4.5 \mathrm{~kg} / \mathrm{m}^{2}$ ) increase in BMI. The 95\% CIs from both of the methods do not include value 1 implying that the estimated positive causal effect of BMI on the risk of heart attacks is unlikely to have occurred due to chance alone.

\section{Discussion}

\section{Surrounding research}

Our two-sample MR analyses showed evidence for a positive causal effect of BMI on the risk of heart attacks. 
Table 1 Summary results of BMI associated independent instrumental SNPS

\begin{tabular}{|c|c|c|c|c|c|c|c|c|c|}
\hline$\overline{S N P}$ & Chr & Position & EA & $\mathrm{OA}$ & EAF & Beta & SE & $P$ & Gene \\
\hline rs1343424 & 1 & $49,376,820$ & $C$ & $T$ & 0.251 & 0.02 & 0.004 & 8.56E-09 & $A G B L 4$ \\
\hline rs2422134 & 1 & $72,514,701$ & C & G & 0.508 & -0.022 & 0.003 & $2.49 \mathrm{E}-13$ & NEGR1 \\
\hline rs6690871 & 1 & $74,977,277$ & G & A & 0.407 & 0.022 & 0.003 & $1.28 \mathrm{E}-12$ & $\angle R R C 53$ \\
\hline rs505066 & 1 & $96,882,671$ & A & C & 0.285 & 0.025 & 0.004 & 2.35E-09 & (UBE2WP1) \\
\hline rs7550711 & 1 & $110,082,886$ & T & C & 0.026 & 0.066 & 0.009 & $9.51 \mathrm{E}-14$ & GPR61 \\
\hline rs4478775 & 1 & $177,770,097$ & A & G & 0.223 & 0.025 & 0.004 & $1.98 \mathrm{E}-11$ & SEC16B \\
\hline rs2820292 & 1 & $201,784,287$ & A & C & 0.432 & -0.02 & 0.003 & $1.58 \mathrm{E}-10$ & NAV1 \\
\hline rs13411762 & 2 & 554,109 & C & T & 0.928 & 0.033 & 0.006 & 5.93E-09 & (TMEM18) \\
\hline rs13391869 & 2 & 606,213 & G & A & 0.848 & -0.022 & 0.004 & $1.65 \mathrm{E}-08$ & (TMEM18) \\
\hline rs10495749 & 2 & $24,730,847$ & A & G & 0.231 & -0.021 & 0.004 & $3.21 \mathrm{E}-09$ & NCOA1 \\
\hline rs11126666 & 2 & $26,928,811$ & G & A & 0.745 & -0.021 & 0.003 & $5.71 \mathrm{E}-10$ & KCNK3 \\
\hline rs13011109 & 2 & $58,857,419$ & G & C & 0.612 & 0.018 & 0.003 & 6.84E-09 & FANCL \\
\hline rs6545714 & 2 & $59,307,725$ & G & A & 0.399 & 0.018 & 0.003 & 3.19E-09 & (FANCL) \\
\hline rs2890652 & 2 & $142,959,931$ & T & C & 0.839 & -0.028 & 0.005 & $2.24 \mathrm{E}-08$ & $(L R P 1 B)$ \\
\hline rs1528435 & 2 & $181,550,962$ & T & C & 0.62 & 0.018 & 0.003 & 4.68E-09 & (UBE2E3) \\
\hline rs7599312 & 2 & $213,413,231$ & G & A & 0.732 & 0.022 & 0.003 & $4.88 \mathrm{E}-11$ & (ERBB4) \\
\hline rs10510554 & 3 & $25,099,776$ & T & C & 0.431 & -0.018 & 0.003 & $1.78 \mathrm{E}-09$ & $R A R B$ \\
\hline rs1916801 & 3 & $61,187,046$ & A & T & 0.595 & 0.019 & 0.003 & 8.13E-10 & FHIT \\
\hline rs3849570 & 3 & $81,792,112$ & A & C & 0.348 & 0.019 & 0.003 & $1.61 \mathrm{E}-08$ & GBE1 \\
\hline rs10511073 & 3 & $85,653,460$ & G & A & 0.646 & -0.021 & 0.003 & $1.46 \mathrm{E}-10$ & CADM2 \\
\hline rs16851483 & 3 & $141,275,436$ & G & T & 0.934 & -0.048 & 0.008 & $1.77 \mathrm{E}-10$ & RASA2 \\
\hline rs4494964 & 3 & $185,776,491$ & T & C & 0.142 & -0.04 & 0.005 & $1.39 \mathrm{E}-15$ & ETV5 \\
\hline rs13144044 & 4 & $45,082,236$ & C & G & 0.534 & -0.025 & 0.004 & $1.02 \mathrm{E}-11$ & (GNPDA2) \\
\hline rs17001654 & 4 & $77,129,568$ & C & G & 0.852 & -0.031 & 0.005 & 3.88E-09 & SCARB2 \\
\hline rs13107325 & 4 & $103,188,709$ & C & $\mathrm{T}$ & 0.925 & -0.048 & 0.007 & 1.15E-12 & SLC39A8 \\
\hline rs11727676 & 4 & $145,659,064$ & C & $\mathrm{T}$ & 0.096 & -0.036 & 0.006 & 1.11E-08 & HHIP \\
\hline rs6871667 & 5 & $74,604,742$ & A & G & 0.401 & -0.017 & 0.003 & $1.44 \mathrm{E}-08$ & (HMGCR) \\
\hline rs280275 & 6 & $50,668,612$ & A & G & 0.952 & -0.039 & 0.007 & 4.46E-09 & (TFAP2D) \\
\hline rs4141973 & 6 & $50,946,521$ & T & C & 0.134 & 0.028 & 0.005 & $5.51 \mathrm{E}-09$ & (FTH1P5) \\
\hline rs9400239 & 6 & $108,977,663$ & C & T & 0.706 & 0.019 & 0.003 & 6.10E-09 & $\mathrm{FOXO3}$ \\
\hline rs13191362 & 6 & $163,033,350$ & A & G & 0.875 & 0.028 & 0.005 & 3.94E-09 & PARK2 \\
\hline rs1167827 & 7 & $75,163,169$ & A & G & 0.435 & -0.02 & 0.003 & 4.64E-10 & HIP1 \\
\hline rs2245368 & 7 & $76,608,143$ & T & C & 0.833 & -0.032 & 0.006 & 1.34E-08 & $D T \times 2 P 1$ \\
\hline rs2922763 & 8 & $76,573,711$ & G & T & 0.274 & -0.024 & 0.004 & $2.73 \mathrm{E}-10$ & (HNF4G) \\
\hline rs2033732 & 8 & $85,079,709$ & $C$ & T & 0.744 & 0.019 & 0.004 & $2.06 \mathrm{E}-08$ & (RALYL) \\
\hline rs4740619 & 9 & $15,634,326$ & T & C & 0.551 & 0.018 & 0.003 & 3.87E-09 & CCDC171 \\
\hline rs1412234 & 9 & $28,410,683$ & C & T & 0.33 & 0.024 & 0.004 & 4.97E-09 & LINGOZ \\
\hline rs6477694 & 9 & $111,932,342$ & C & $\mathrm{T}$ & 0.354 & 0.017 & 0.003 & 9.95E-09 & (FRRS1L) \\
\hline rs1928295 & 9 & $120,378,483$ & C & T & 0.43 & -0.019 & 0.003 & $6.62 \mathrm{E}-10$ & (TLR4) \\
\hline rs10733682 & 9 & $129,460,914$ & A & G & 0.473 & 0.017 & 0.003 & 9.95E-09 & $L M X 1 B$ \\
\hline rs7899106 & 10 & $87,410,904$ & A & G & 0.95 & -0.04 & 0.007 & 1.32E-08 & GRID1 \\
\hline rs17094222 & 10 & $102,395,440$ & C & T & 0.213 & 0.025 & 0.004 & $2.83 \mathrm{E}-11$ & $(P A X 2)$ \\
\hline rs17747324 & 10 & $114,752,503$ & C & T & 0.227 & -0.023 & 0.004 & 1.16E-09 & TCF7L2 \\
\hline rs1374262 & 11 & $8,458,749$ & G & A & 0.532 & 0.017 & 0.003 & 1.44E-08 & STK33 \\
\hline
\end{tabular}


Table 1 Summary results of BMI associated independent instrumental SNPs (Continued)

\begin{tabular}{|c|c|c|c|c|c|c|c|c|c|}
\hline SNP & Chr & Position & EA & OA & EAF & Beta & SE & $P$ & Gene \\
\hline rs4514364 & 11 & $27,456,059$ & $C$ & $\mathrm{~T}$ & 0.316 & 0.02 & 0.003 & 1.72E-09 & LGR4 \\
\hline rs2176598 & 11 & $43,864,278$ & T & $C$ & 0.246 & 0.02 & 0.004 & 1.90E-08 & HSD $17 B 12$ \\
\hline rs2856650 & 11 & $47,365,199$ & G & $A$ & 0.703 & -0.02 & 0.003 & 9.87E-10 & MYBPC3 \\
\hline rs12286929 & 11 & $115,022,404$ & G & A & 0.527 & 0.022 & 0.003 & $1.28 \mathrm{E}-12$ & (CADM1) \\
\hline rs2720296 & 12 & $50,169,070$ & G & A & 0.613 & -0.02 & 0.003 & $9.87 \mathrm{E}-10$ & (LSM6P2) \\
\hline rs11057405 & 12 & $122,781,897$ & A & G & 0.106 & -0.031 & 0.006 & 1.19E-08 & CLIP1 \\
\hline rs7988412 & 13 & $28,000,282$ & $C$ & $\mathrm{~T}$ & 0.815 & -0.026 & 0.005 & 1.10E-08 & GTF3A \\
\hline rs2797084 & 13 & $54,071,824$ & T & $C$ & 0.86 & -0.032 & 0.006 & $6.12 \mathrm{E}-09$ & (OLFM4) \\
\hline rs10132280 & 14 & $25,928,179$ & A & C & 0.3 & -0.023 & 0.003 & $6.68 \mathrm{E}-12$ & (STXBP6) \\
\hline rs2370982 & 14 & $79,890,677$ & T & $C$ & 0.216 & 0.025 & 0.005 & 2.43E-08 & NRXN3 \\
\hline rs3736485 & 15 & $51,748,610$ & A & G & 0.461 & 0.018 & 0.003 & 6.84E-09 & DMXL2 \\
\hline rs12905371 & 15 & $67,845,930$ & $C$ & $\mathrm{~T}$ & 0.736 & 0.021 & 0.004 & 4.46E-09 & MAP 2 K5 \\
\hline rs12448257 & 16 & $3,599,655$ & G & A & 0.784 & -0.024 & 0.004 & $8.42 \mathrm{E}-10$ & NLRC3 \\
\hline rs2531995 & 16 & $4,013,467$ & $C$ & $T$ & 0.379 & -0.024 & 0.004 & 8.46E-10 & ADCY9 \\
\hline rs2354584 & 16 & $19,778,575$ & A & G & 0.87 & 0.035 & 0.006 & 1.10E-09 & IQCK \\
\hline rs2650492 & 16 & $28,333,411$ & A & G & 0.298 & 0.021 & 0.004 & 1.67E-09 & SBK1 \\
\hline rs2303222 & 16 & $31,085,470$ & $C$ & $\mathrm{~T}$ & 0.378 & -0.018 & 0.003 & 1.78E-09 & ZNF668 \\
\hline rs1477199 & 16 & $53,712,135$ & A & G & 0.864 & -0.024 & 0.004 & 1.90E-08 & RPGRIPIL \\
\hline rs7205986 & 16 & $53,755,146$ & G & $A$ & 0.511 & 0.025 & 0.003 & $3.68 \mathrm{E}-16$ & FTO \\
\hline rs3026101 & 17 & $5,280,440$ & $C$ & $\mathrm{~T}$ & 0.301 & 0.018 & 0.003 & 1.23E-08 & NUP88 \\
\hline rs12949279 & 17 & $78,558,411$ & $C$ & $T$ & 0.439 & -0.018 & 0.003 & 8.25E-09 & RPTOR \\
\hline rs891389 & 18 & $21,087,531$ & $C$ & $\mathrm{~T}$ & 0.655 & -0.021 & 0.004 & $2.20 \mathrm{E}-08$ & RMC1 \\
\hline rs7243357 & 18 & $56,883,319$ & G & $T$ & 0.176 & -0.022 & 0.004 & 2.90E-08 & (GRP) \\
\hline rs4940929 & 18 & $57,803,890$ & G & C & 0.583 & 0.027 & 0.004 & $3.43 \mathrm{E}-11$ & (MC4R) \\
\hline rs9944545 & 18 & $57,958,244$ & T & $C$ & 0.297 & 0.034 & 0.003 & $5.66 \mathrm{E}-24$ & (MC4R) \\
\hline rs17724992 & 19 & $18,454,825$ & A & G & 0.733 & 0.019 & 0.004 & 1.49E-08 & PGPEP1 \\
\hline rs29944 & 19 & $34,306,898$ & A & G & 0.327 & -0.018 & 0.003 & 2.07E-08 & (KCTD15) \\
\hline rs2075650 & 19 & $45,395,619$ & $\underline{A}$ & $\underline{G}$ & 0.854 & $\underline{0.026}$ & $\underline{0.005}$ & 4.92E-09 & TOMM40 \\
\hline rs9304665 & 19 & $47,602,577$ & A & $\mathrm{T}$ & 0.765 & 0.025 & 0.004 & 7.61E-09 & $\mathrm{ZC} 3 \mathrm{H}_{4}$ \\
\hline
\end{tabular}

Table 1 lists summary results of the 73 independent SNPs used as instruments in our MR analysis. These SNPs were associated with BMI $\left(P<5 \times 10^{-8}\right)$ in the European adults in the GIANT study. One SNP rs2075650 appeared to be an outlier. We re-did MR analysis without this SNP. SNP: single nucleotide polymorphism, Chr: chromosome, Position: location of the SNP in the chromosome according to Build 37, EA: effect allele, OA: the other allele, EAF: effect allele frequency. Beta, SE and P are estimated SNP effect, standard error and $p$-value from the GIANT GWAS study. Gene: genes of the SNPs located in or close to. For intergenic SNPs, their nearest genes are provided in brackets

There are many previous studies that have used MR and reported a causal link between BMI and cardiovascular diseases $[3,27,28]$. To the best of our knowledge, however, this current study is the first to use MR to explore if increased BMI causes an increase specifically in the risk of heart attacks. This is important as the root causes of cardiovascular diseases as a whole are generally too broad to find the biological reasoning behind the mechanisms. Choosing a smaller target makes interpretation of these mechanisms much clearer.

This new evidence aids the growing theory that obesity is associated with a heightened risk of a plethora of cardiac related disease phenotypes. Such studies that have also contributed to this theory include one which linked BMI to heart failure using the ENGAGE consortium $(n=19,384)$ [29], and another which used the UK Biobank to link BMI to aortic valve stenosis $(n=367$, 703) [27]. The latter study also related BMI to a lot of other cardiovascular diseases and so came the closest to detailing the causal effect of BMI on heart attacks although they did not explicitly state this. Furthermore, the BMI data they used was sourced elsewhere to GIANT. Overall, it seems that much of existing research does support similar evidence that BMI is related to a large array of heart disease, including heart attacks. 

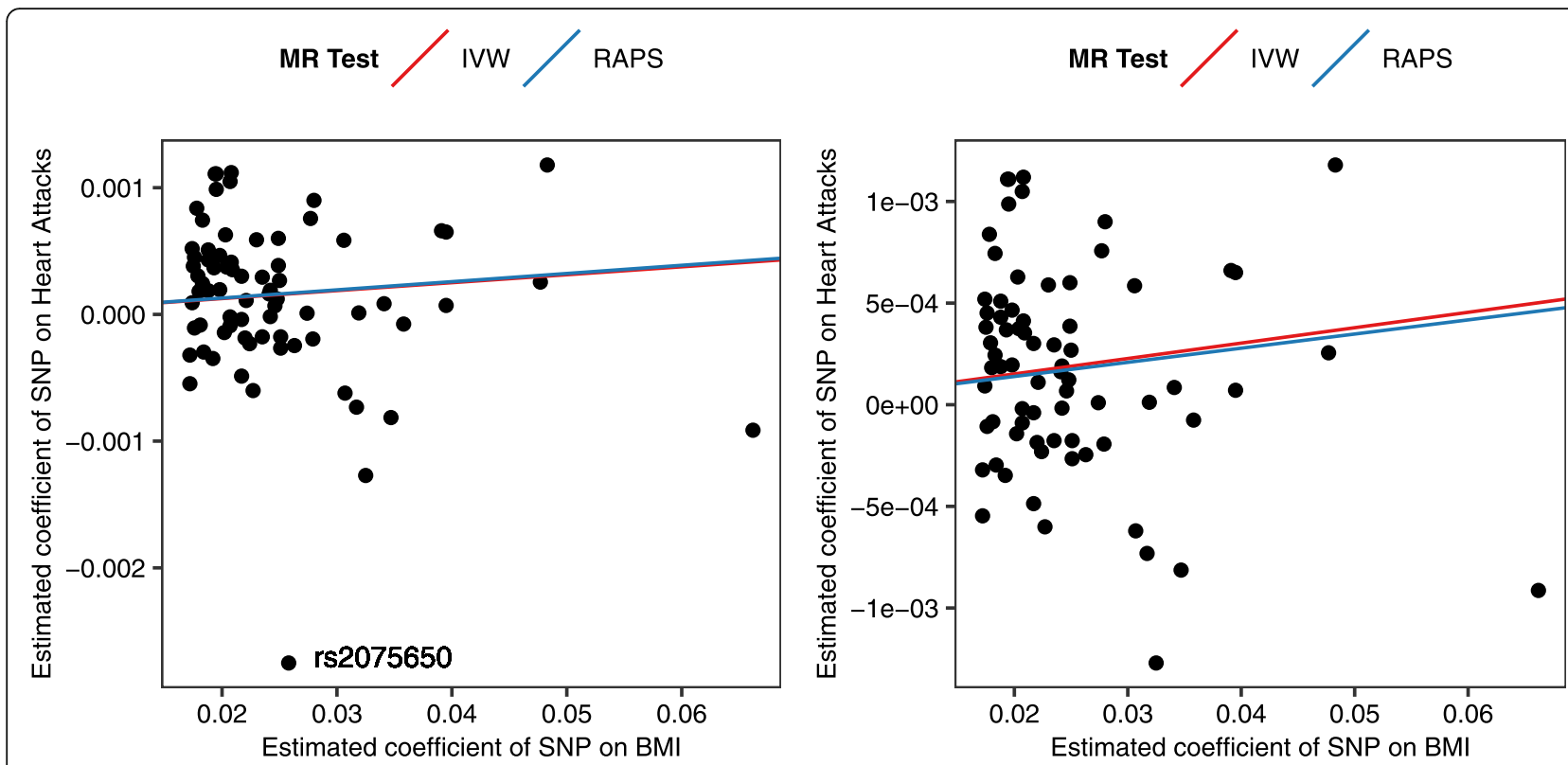

Fig. 2 MR results with (left panel) and without (right panel) outlier SNP rs2075650. Each dot, corresponding to one of the instrumental SNPs of the investigation, represents estimated regression coefficient of the SNP on BMI (horizontal axis) and on heart attacks (vertical axis). The slopes are causal effects (log odds ratios) of BMI on heart attacks estimated from two MR methods: inverse variance weighted (IVW) estimation (red) and robust adjusted profile score (RAPS) (blue)

\section{Biological mechanism}

High BMI values have been found to cause hypertension through MR tests [30, 31]. These results were reported to be independent of age, sex or confounders such as smoking and alcohol intake. Hypertension adds force onto the arterial walls as blood flows through. Continued exposure to these larger forces increases the chances of arterial wall damage and the accumulation of cholesterol, other lipids and therefore plaques. Plaques can narrow the diameter of the arteries that provide oxygenated blood to the heart muscle. This is usually asymptomatic but cases of angina or other chest pains are common, especially when one is exerting themselves. Eventually, the plaque will either grow to fully occlude the lumen of the artery or potentially break off to block a narrower section downstream of the plaque. In both instances, blood flow is cut off to the heart and so the individual experiences a heart attack (myocardial infarction) which can be fatal.

Elevated BMI has also been associated with increased blood interleukin 6 (IL-6) and expression of its receptor IL-6R [6]. IL-6 has been linked to the development of

Table 2 Estimated causal effect of BMI on heart attacks from two MR methods

\begin{tabular}{llll}
\hline MR Method & Estimated OR & $95 \% \mathrm{Cl}$ & $P$-value \\
\hline IVW & 1.008 & $(1.003,1.012)$ & 0.001 \\
RAPS & 1.007 & $(1.002,1.012)$ & 0.004 \\
\hline
\end{tabular}

atherosclerotic plaques through increasing the rate of synthesis of its components. Lipid concentrations, blood glucose, C-reactive protein (CRP), interleukin 18 and adiponectin are also commonly used as markers for heart attacks and have been shown to be increased in the obese [32].

Though this is not an exhaustive list of the potential biological mechanisms behind the causal association between obesity and heart attacks, they are some of the most common causes in the UK population. It is important to note that just because this association is found through genetic instruments, it does not mean that lifestyle changes cannot alter the chances of a heart attack outcome. Regular exercise and a low-fat diet, along with weight loss have been shown to reduce the chances of heart attacks [32].

\section{Strengths \& Limitations}

Using UK Biobank and GIANT was advantageous for our investigation due to their large sample size and high quality of data. Focusing only on European ancestry and imputed SNPs with high accuracy mitigated primary confounding factors e.g. population structure and imputation errors.

MR is the star attraction of this investigation. It has allowed us to investigate the causal association of BMI on heart attacks without the usual pitfalls of confounders being an issue. The MR design has many strengths as it relies solely on genetic instruments. 
Alleles are randomly sorted and become fixed at conception and MR then directly tests these alleles, which bypasses biases due to confounding and reverse causality.

A limitation very common to MR studies is pleiotropy, and this study is no exception. Pleiotropy was the reason for the removal of SNP rs2075650 due to the belief that the SNP was also associated with Alzheimer's disease. The use of a robust method RAPS means that our analysis was improved by reducing the likelihood of biased results due to weak instruments.

A limitation of our study was the terminology used in the UK Biobank data with reference to the outcome. The term 'heart attack' can be open to interpretation when it comes to what actually went wrong in the body. Not only because there are five recognised forms of heart attacks [33], but also because to a member of the public answering the UK Biobank questionnaires, they may believe their coronary thrombosis to be a heart attack, when in fact in this investigation we assumed by heart attack, the participant was referring to a myocardial infarction, which while similar in its outcome, has different causes.

\section{Conclusions}

Upon conducting MR analysis on European descendants, this investigation has shown some evidence that a higher BMI causes an increased risk of an individual having a heart attack. Therefore, individuals who are overweight should look to reduce their weight and health services should look to producing guidelines that encourage this behaviour.

These findings suggest that parameters should be put in place following consultations with obese and overweight patients that look to induce their weight loss as we now know that it may have a direct causal association with heart attacks. Such parameters could be an automatic appointment with a dietitian or a leaflet on local places to exercise. Should future GWAS re-impute the same data, their questionnaires on heart attacks should contain more specific terminology (i.e. instead of asking whether the patient has had a heart attack, they could ask which type of myocardial infarction they have had). Then MR analysis could be carried out on BMI's causal association with each type of myocardial infarction. This could help with reducing wasting the aforementioned parameters on patients who potentially would not benefit from them. Future research could also focus on the action of the proteins the identified SNPs are related to and on understanding their function in the body.

\section{Abbreviations}

GIANT: Genetic investigation of anthropometric traits; MR: Mendelian randomisation; SNP: Single nucleotide polymorphism; INW: Inverse variance weighted estimation; OR: Odds ratio; SD: Standard deviation; Cl: Confidence interval; RAPS: Robust adjusted profile score; GWAS: Genome wide association study; LD: Linkage disequilibrium; QC: Quality control; MAF: Minor allele frequency; HWE: Hardy-Weinberg equilibrium; TOMM40: Translocase of outer mitochondrial membrane 40; APOE: Apolipoprotein E;

ENGAGE: European network for genetic and genomic epidemiology; IL6(R): Interleukin 6 (receptor); CRP: C-reactive protein

\section{Acknowledgements}

We thank the GIANT consortium and the Neale lab for making GWAS results publicly accessible.

\section{Authors' contributions}

$H G$ and BA conceived and designed the study. BA and $L$ performed statistical analysis. HG and BA interpreted statistical results. BA, HG and LJ wrote the manuscript. All authors read and approved the final manuscript.

\section{Funding}

This work was funded by the Medical Research Council (grant number MR/ N013751/1). The funding body had no role in the study design, data collection and analysis, interpretation of data and preparation of the manuscript.

Availability of data and materials

Not applicable.

\section{Ethics approval and consent to participate}

In this study, we only used summary results from previous GWASs that are publicly accessible. Ethics approval and consent to participate were available in the GIANT and UK Biobank studies.

Consent for publication

Not applicable.

\section{Competing interests}

The authors declare that they have no competing interests.

Received: 6 March 2020 Accepted: 20 May 2020

Published online: 29 May 2020

\section{References}

1. Baker C. Obesity statistics. 2019. https://researchbriefings.parliament.uk/ ResearchBriefing/Summary/SN03336. Accessed 19 Aug 2019.

2. Atlantis $\mathrm{E}$, Baker M. Obesity effects on depression: systematic review of epidemiological studies. Int J Obes. 2008:32:881-91.

3. Zhang X, Lv WQ, Qiu B, Zhang LJ, Qin J, Tang FJ, et al. Assessing causal estimates of the association of obesity-related traits with coronary artery disease using a Mendelian randomization approach. Sci Rep. 2018;8:7146.

4. Buitrago F, Calvo Jl, Redondo-López V, Canón-Barroso L, Rodríguez-Pérez L, Hinojosa-Díaz JF. Cardiovascular events in patients with obesity: an observational study. Br J Gen Pract. 2010;60:584-9.

5. Tsai F, Coyle WJ. The microbiome and obesity: is obesity linked to our gut flora? Curr Gastroenterol Rep. 2009:11:307-13.

6. Sindhu S, Thomas R, Shihab P, Sriraman D, Behbehani K, Ahmad R. Obesity is a positive modulator of IL-6R and IL-6 expression in the subcutaneous adipose tissue: significance for metabolic inflammation. PLoS One. 2015;10: e0133494.

7. John S. Deaths registered in England and Wales - Office for National Statistics. Office for National Statistics 2019. https://www.ons.gov.uk/ peoplepopulationandcommunity/birthsdeathsandmarriages/deaths/ bulletins/deathsregistrationsummarytables/2018\#leading-causes-of-death. Accessed 19 Aug 2019

8. Mittendorfer B, Peterson LR. Cardiovascular consequences of obesity and targets for treatment. Drug Discov Today Ther Strateg. 2008;5:53-61.

9. Shahar E. The association of body mass index with health outcomes: causal, inconsistent, or confounded? Am J Epidemiol. 2009:170:957-8.

10. Greenberg JA. Correcting biases in estimates of mortality attributable to obesity. Obesity. 2006:14:2071-9.

11. University of Bristol. Evidence that BMI has an independent and causal effect on heart disease risk. Science Daily. 2012. https://www.sciencedaily. com/releases/2012/05/120501183017.htm. Accessed 12 Aug 2019. 
12. Stallones RA. The association between tobacco smoking and coronary heart disease. Int J Epidemiol. 2015;44:735-43.

13. Mukamal K, Lazo M. Alcohol and cardiovascular disease. BMJ. 2017;356: j1340.

14. Burgess S, Davies NM, Thompson SG. Bias due to participant overlap in twosample Mendelian randomization. Genet Epidemiol. 2016;40:597-608.

15. Locke AE, Kahali B, Berndt SI, Justice AE, Pers TH, Day FR, et al. Genetic studies of body mass index yield new insights for obesity biology. Nature. 2015;518:197-206.

16. The Neale Lab. UK Biobank GWAS Results. 2018. http://www.nealelab.is/ukbiobank. Accessed 9 Nov 2019.

17. Hemani G, Zheng J, Elsworth B, Wade KH, Haberland V, Baird D, et al. The MR-base platform supports systematic causal inference across the human phenome. Elife. 2018;7:e34408.

18. UK Biobank. https://www.ukbiobank.ac.uk/about-biobank-uk/. Accessed 19 Feb 2020.

19. UK Bioank. Data-Field 6150. http://biobank.ctsu.ox.ac.uk/crystal/field.cgi?id= 6150. Accessed 14 Aug 2019

20. Bowden J, Smith GD, Burgess S. Mendelian randomization with invalid instruments: effect estimation and bias detection through egger regression. Int J Epidemiol. 2015.

21. Zhao Q, Wang J, Hemani G, Bowden J, Small DS. Statistical inference in twosample summary-data Mendelian randomization using robust adjusted profile score; 2018

22. Burgess S, Butterworth A, Thompson SG. Mendelian randomization analysis with multiple genetic variants using summarized data. Genet Epidemiol. 2013;37:658-65.

23. Hemani G, Bowden J, Davey SG. Evaluating the potential role of pleiotropy in Mendelian randomization studies. Hum Mol Genet. 2018:R195-208.

24. Ensembl Database. rs2075650 (SNP) - Phenotype Data - Homo sapiens GRCh37. http://grch37.ensembl.org/Homo_sapiens/Variation/ Phenotype?db=core; $r=19: 45395119-4539611$ 9; $v=r s 2075650 ; v d b=v a r i a t i o n ; v f=$ 584400527. Accessed 8 Aug 2019.

25. The National Center for Biotechnology Information (NCBI). Reports of genes. https://www.ncbi.nlm.nih.gov/gene/. Accessed 29 Apr 2020.

26. Lyall DM, Harris SE, Bastin ME, Muñoz Maniega S, Murray C, Lutz MW, et al. Alzheimer's disease susceptibility genes APOE and TOMM40, and brain white matter integrity in the Lothian Birth Cohort 1936. Neurobiol Aging. 2014;35:1513.e25-33

27. Larsson SC, Bäck M, Rees JMB, Mason AM, Burgess S. Body mass index and body composition in relation to 14 cardiovascular conditions in UK biobank: a Mendelian randomization study. Eur Heart J. 2020;41:221-6.

28. He L, Culminskaya I, Loika Y, Arbeev KG, Bagley O, Duan M, et al. Causal effects of cardiovascular risk factors on onset of major age-related diseases: a time-to-event Mendelian randomization study. Exp Gerontol. 2018;107:7486.

29. Hägg S, Fall T, Ploner A, Mägi R, Fischer K, Draisma HHM, et al. Adiposity as a cause of cardiovascular disease: a Mendelian randomization study. Int J Epidemiol. 2015;44:578-86.

30. Lyall DM, Celis-Morales C, Ward J, lliodromiti S, Anderson JJ, Gill JMR, et al. Association of body mass index with cardiometabolic disease in the UK biobank: a mendelian randomization study. JAMA Cardiol. 2017;2:882-9.

31. Dale CE, Fatemifar G, Palmer TM, White J, Prieto-Merino D, Zabaneh D, et al. Causal associations of adiposity and body fat distribution with coronary heart disease, stroke subtypes, and type 2 diabetes mellitus: a Mendelian randomization analysis. Circulation. 2017;135:2373-88.

32. Esposito K, Pontillo A, Di Palo C, Giugliano G, Masella M, Marfella R, et al. Effect of weight loss and lifestyle changes on vascular inflammatory markers in obese women: a randomized trial. J Am Med Assoc. 2003;289:1799-804.

33. Chapman AR, Adamson PD, Mills NL. Assessment and classification of patients with myocardial injury and infarction in clinical practice. Heart. 2017;103:10-8.

\section{Publisher's Note}

Springer Nature remains neutral with regard to jurisdictional claims in published maps and institutional affiliations.

\section{Ready to submit your research? Choose BMC and benefit from:}

- fast, convenient online submission

- thorough peer review by experienced researchers in your field

- rapid publication on acceptance

- support for research data, including large and complex data types

- gold Open Access which fosters wider collaboration and increased citations

- maximum visibility for your research: over $100 \mathrm{M}$ website views per year

At BMC, research is always in progress.

Learn more biomedcentral.com/submissions 